\title{
IDO-1 Inhibitor LY3381916
}

National Cancer Institute

\section{Source}

National Cancer Institute. IDO-1 Inhibitor LY3381916. NCI Thesaurus. Code C143068.

An orally available inhibitor of indoleamine 2,3-dioxygenase 1 (IDO1; IDO-1), with potential immunomodulating and antineoplastic activities. Upon administration, IDO1 inhibitor LY3381916 specifically targets and binds to IDO1, a cytosolic enzyme responsible for the oxidation of the amino acid tryptophan into the immunosuppressive metabolite kynurenine. By inhibiting IDO1 and decreasing kynurenine in tumor cells, LY3381916 restores and promotes the proliferation and activation of various immune cells, including dendritic cells (DCs), natural killer (NK) cells, and T-lymphocytes, and causes a reduction in tumor-associated regulatory T-cells (Tregs). Activation of the immune system, which is suppressed in many cancers, may induce a cytotoxic T-lymphocyte (CTL) response against the IDO1-expressing tumor cells, thereby inhibiting the growth of IDO1expressing tumor cells. IDO1, overexpressed by multiple tumor cell types, plays an important role in immunosuppression. Tryptophan depletion inhibits T-lymphocyte proliferation and activation, and subsequently suppresses the immune system. 